http://jmscr.igmpublication.org/home/

ISSN (e)-2347-176x ISSN (p) 2455-0450

crossref DOI: https://dx.doi.org/10.18535/jmscr/v9i6.08

Journal Of Medical Science And Clinical Research

\title{
A Comparative Study on Diabetes (Diabetes Mellitus) (Metabolic Disease that occurs when an Individual Has Elevated Blood Glucose)
}

\author{
Authors \\ Rahul Kumar ${ }^{1}$, Rajeev Ranjan Kumar ${ }^{2^{*}}$ \\ Senior Residents, Department of Medicine, AIIMS -Patna, India \\ *Corresponding Author \\ Rajeev Ranjan Kumar
}

\begin{abstract}
Backdrop and Objectives: Diabetes is also known as: diabetes mellitus (DM), it can cause many serious problems, it is not diagnosed at the time and it can also lead to death in several cases. it. Is the bundle of several metabolic levels which is over hypothyroidism he very long time period. their is a universal symbol for diabetes as shown in figure 1.

Description: 463 million people have diabetes in this world.88 million people with diabetes are in southeast Asia region .88 million people, 77 with diabetes are in India so the prevalence of the population is $8.9 \%$, according to the IDF.

The majority living in low-and middle-income countries, and 1.6 million of people died by diabetes each year.
\end{abstract}

\section{Introduction}

\section{Types of diabetes}

- The autoimmune disease which is the Type 1 diabetes. where insulin is made, the immune system attacks and damaged the cells in the pancreas.

- When body is able to have the power of resistance, to the substance called insulin, the glucose (sugar) built up in the blood, giving it the name that is Type 2 diabetes.

- When the blood glucose ,that is when the blood sugar is very high more than its moderate level, but not high as it's possible to diagnose or checking, for the type 2 diabetes, the condition is called Prediabetes.
- When the women is going through the period of maternity, the pregnancy ,the insulin - blocking hormones developed by the placenta causes the a very high blood sugar to be known as Gestational Diabetes.

Each one of these diabetes has unique symptoms, causes and a different types of medical treatments.

\section{Results}

The results include the various symptoms of diabetes including the following:

- Rise in the Hunger of a person

- Rise in the water consumption

- The loss in the person's weight

- The very frequent urination

- The eyes see's everything blurry 
- The extreme level of fatigue, weakness

- Sores which do not heal at all Although there are several skin problems caused by diabetes as shown in the figure 2,3and 4 .

\section{Eruptive xanthomatosis}

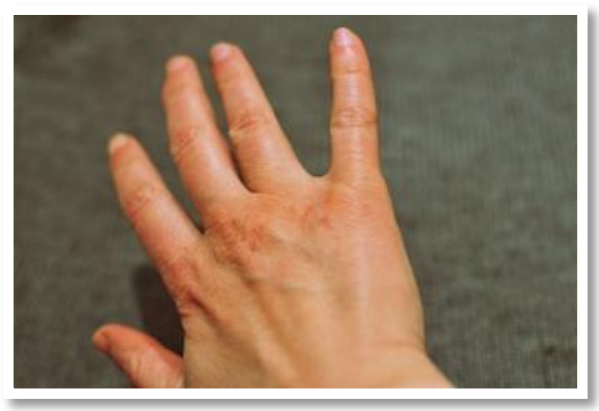

Figure 1

\section{Bacterial infections}

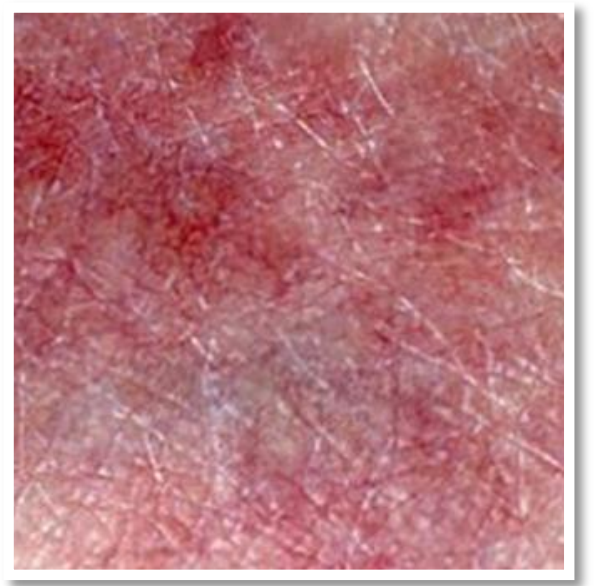

Figure 2

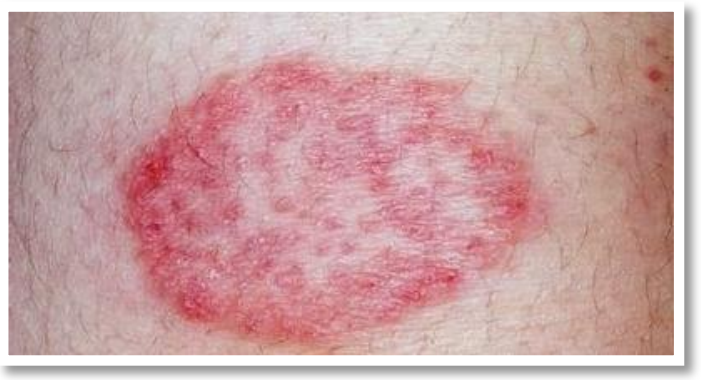

\section{Diagnosis/procedure}

There is a blood test called GLYCATED HEMOGLOBIN THAT IS(A1C) TEST, which do not have a important requirement that is to fasting, which indicates the average blood sugar which a person is having from a last two to three months. The percentage of the sugar which is attached to the person's haemoglobin, is measured. The sugar levels are attached to the more haemoglobin, the person is having as. It is directly proportionate. An
AIC level of $6.5 \%$ or higher than this on the two of the different test, the person is having diabetes. The doctor may prescribe the common test as follows

- Random blood sugar test- This test is suggested by it's name itself, it is done randomly, anytime without seeing the condition like, when did you last ate, blood sugar level.

- Fasting blood sugar Test- In this test the sugar level which is normal than (5.6 $\mathrm{mmol} / \mathrm{L}$ ). This test is done only after the person haven't eaten something the overnight till the day of the test.

- Oral glucose tolerance test -This is the test in which the person have to fast overnight, And the fasting fasting blood sugar level is measured. The person is than allowed to drink a sugary liquid, and the periodically, the blood sugar level is tested for the upcoming next two hours.

\section{There are also some screening test as followed}

- Initial glucose challenge test

In the beginning, the person is given the sugary syrup drinking for the glucose challenge test. After 1 hour, the person's blood test is done as in which the level below $149 \mathrm{mg} / \mathrm{dL}$ is usually mentioned as a normal on this test, but it varies at a specific clinical area's and labs.

If the person's blood sugar level is higher than the normal rating in this test, that means the person is at risk of having gestational diabetes.

\section{- Follow-up glucose tolerance testing}

In this test the person is said to have a fast overnight, so that the test can be done. After the fast, the next day the test is done that is the blood test, than is person is given the sweet solution to drink, but this it is more concentrated with glucose.

After this the person's blood sugar is checked again and again for every hour for a period of there hours. 
For being diagnosed with gestational diabetes, at least the two of the blood sugar readings should be higher than the normal values established for each of the three hours.

\section{Treatment/End result}

There are several Ways of treating the diabetes, diet and exercise are very much good and enough to maintain and keep the disease under control. Other people with very complications, needs medication, which includes insulin and oral drug, it works in a very different way and brings blood sugar level back to the normal condition. There are best food to control Diabetes such as fatty fish, some people consider fatty fish to be the healthiest food, leafy grey, Avocados, Eggs, Chia seeds, Beans, Geek yogurt ,Nuts and many more.

\section{Conclusion}

Diabetes is very dangerous especially for the old people, diabetes causes many several problems. The chest pain is also caused by. The diabetes. The cardiovascular problems are also associated with the diabetes. The heart attack, Stroke, is also caused by diabetes.

The narrowing of the arteries that is known as Atherosclerosis, is also the sign or we can say is also caused by the diabetes, so the diet should be conscious.

The nervous system is damaged leading to neuropathy. Proper medical treatment is required for diabetes. The patient have to be conscious towards his/her diet, the medicines should be given at proper time.

\section{Important Note}

In Severe Cases, The diabetes may leads to death if the patient is not getting a proper treatment and proper medicines at proper time.

\section{References}

1. Centres for Disease Control and Prevention. National Diabetes Statistics Report: Estimates of Diabetes and it's burden in the United States, 2014. Atlanta,
GA: U.S. Department of Health and Human Services; 2014.[2015 February 26].

2. Lee JW, Brancati FL, yeh HC. Trends in the prevalence of type 2 diabetes in Asians versus White's: results from the United States National Health Interview survey, 1997-2008.Diabetes care.2011 feb ; 34(2);35-7.

3. Maruthur NM. the GROWING prevalence of type 2. Diabetes increased incidence of improved survival? Curr Diab Rep.2013 Dec;(6):786-94.

4. Centre's for disease control and prevention. Diabetes Public Health Resource: Incidence and Age at Diagnosis. 2013 [2015 January 217].

5. Anon standards of medical care in diabetes -2014.Diabtes Care .2014;37 (SUPPL.1):S14-S89.

6. Anon. Economic costs of diabetes in the U.S. In 2006. Diabetes care 2008 Mar;31(3):596-615.

7. Anon. Intensive blood -glucose control with sulphonylureas or insulin compared with conventional treatment and risks of complications in patients with type b2 diabetes (UKPDS 33).UK Prospective Diabetes Study (UKPDS) Lancet.1998 Sep 12;352(9131):837-53.

8. Duckworth W, Abraira C, Mortiz T,et al . Glucose control and vascular complications in veterans' with type 2 diabetes. The. New England journal of medicine.2009 jan8;360(2):119-39.

9. Gerstein HC, Miller ME, Byington RP,et al. Effects of intensive glucose lowering in type 2 diabetes, The New England journal if medicine.2008 Jan 12;358(24):2545-59.

10. Papademetrioub V, Lovato L, Doumas M, et al. Chronic kidney disease and intensive glycaemic control increase cardiovascular risk in patients with type 2 diabetes. Kidney Int. 2014sep 17. 